\title{
MOUSE ON FINGER TIPS USING ML AND AI
}

\author{
Prof .Pallavi Gulve, Nilesh Patil, Gaurav Sali, Nikhil Lokhande \\ BE (CSE), JSPM's BSIOTR, \\ Wagholi, Pune
}

\begin{abstract}
- several modules are created to assist the original world act with the computer world. Here we tend to gift a completely unique way to approach for Human Computer Interaction (HCI) wherever, we tend to management indicator movement employing a period web camera and color code pointers. Our technique is to use a web camera and computer vision method, like framing video frame, video frame segmentation, background removal and color pursuit, to manage mouse tasks (single clicking, open clicking, double-clicking and scrolling actions) and that we show however it will perform everything as ordinary mouse devices will. A color pointer has been used for the action recognition and pursuit, therefore on implement the module with none physical contact with the process. Click events of the mouse are achieved by police work the quantity of tips on the pictures. The appliance has been created in python with software process as windows ten. This technique principally focuses on the proper use of an internet Camera to implement a VR human computer interaction device in an exceedingly in an exceedingly manner.
\end{abstract}

Keywords - Human Computer Communication, Background Removal, Color Finder, Web Camera, computer vision method.

\section{INTRODUCTION}

There's no different a lot of widespread navigation/interaction device than the mouse. Keeping this in mind, we tend to gift a plan of implementing a VR mouse process as another. User makes a specified hand gesture that's captured by a camera.

Object recognition methods area unit wont to extract data from the capture. This can be then translated to some pregnant event on the screen. During this process there's no wire connection between the pc and mouse nor a wireless transmitter-receiver try because the mouse movement's area unit transferred to the pc by the camera.

Gesture is defined as Associate in nursing communicatory, pregnant body motion that expresses a plan, opinion, emotion, etc. Human Communication with a pc within which human gestures, typically hand movements, area unit recognized by the pc webcam. Gesture understanding is employed to act with computers, like deciphering language. The hand gesture recognition technique will be will be 2 types: 3D-hand model approach and also the look primarily based approach. A fix gesture is determined at the explosive burst of your time whereas a variable gesture is meant to vary over an amount of your time. A waving hand means that au revoir is Associate in Nursing example of variable gesture and also the stop sign is Associate in nursing example of fix gesture. To grasp a full message, it's necessary to interpret all the fix and variable gestures over an amount of your time. This advanced method is termed gesture understanding.

\section{OVERVIEW}

Many developer in technology and human computer communication developed varied technologies associated with VR keyboard and mouse. But all of them are using totally different methods.

The various HCI Methods that area unit getting used since earlier times having limit. They block the betterment of computer dependent input devices. It's currently a general tendency to minimize human efforts and overcome the standard, historically getting used computer operated devices. Assuming Associate in Nursing example of a keypad, it's impractical to cut back the complete keypad during a very tiny size so it. will accommodate the fingers. Therefore it becomes somewhat problem whereas operating wherever correctness is required.

Gesture is defined as Associate in nursing communicatory, pregnant body motion that expresses a plan, opinion, emotion, etc. Human Communication with a pc within which human gestures, typically hand movements, area unit recognized by the pc webcam. Gesture understanding is employed to act with computers, like deciphering language. The hand gesture recognition technique will be will be 2 types: 3D-hand model approach and also the look primarily based approach. A fix gesture is determined at the explosive burst of your time whereas a variable gesture is meant to vary over an amount of your time. A waving hand means that au revoir is Associate in Nursing example of variable gesture and also the stop sign is Associate in nursing example of fix gesture. To grasp a full message, it's necessary to interpret all the fix and variable gestures over an amount of your time. This advanced method is termed gesture understanding.

\section{PURPOSE}

The view of the project is to make a VR mouse that works with the assistance of an internet camera. During this project a camera unceasingly takes pictures of color hand movements of 
a user that is then mapped with mouse inputs. This implies that we are able to offer commands to pc while not having any physical communication to the pc and while not having any hardware implementation.

\section{PROBLEM DEFITATION}

To implement a software package resolution to a haul. The primary process is to grasp the matter. The matter here is to create the simplest way so that humans will communicate with a pc while not having any physical communication to the pc. Several ideas were advance however all of them needed physical movement of hardware. That the judicial decision is to create a VR mouse that uses straightforward and low cost video frame process methods

\section{WORKING}

The projected process could be a current video process that's supported a true time process. This may replace one in all the historically used data input device i.e. mouse so just by exploitation the hand gestures the user are able to act naturally with their pc. The fundamental diagram of the projected process is as shown below within the figure.

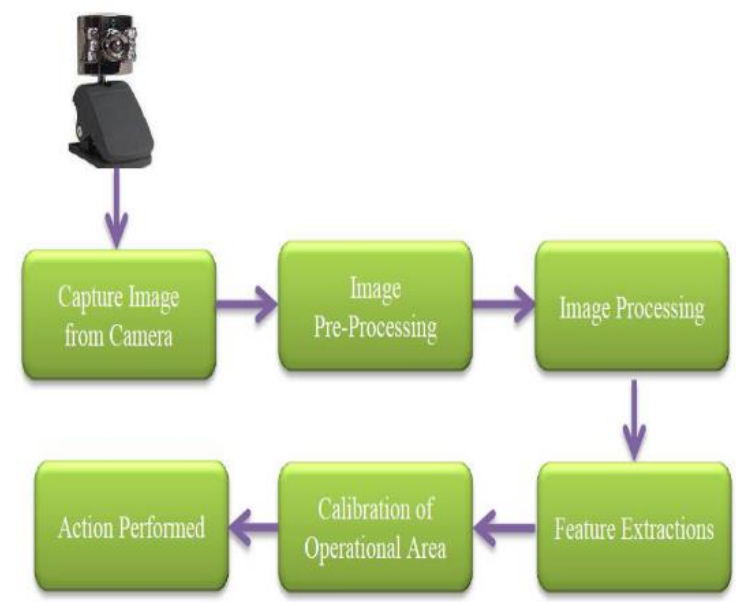

Fig. 1. Single Frame Working Flow

As presently because the color coded fingertips area unit detected by the camera, it starts capturing the pictures the pictures the photographs $\}$ and removes the present video frames and starts process. The processed video frame is given as input to subsequent section of process wherever it starts police work the color coded fingertips. Following the centers and edges. These all extracted options area unit used for the more process like police work hand gestures and dealing in keeping with it. The flow diagram of the projected process on top of is shown for the one frame that is captured. The process could be a real time process that the flow diagram could be a continuous method for each and every frame that's captured by the online camera.

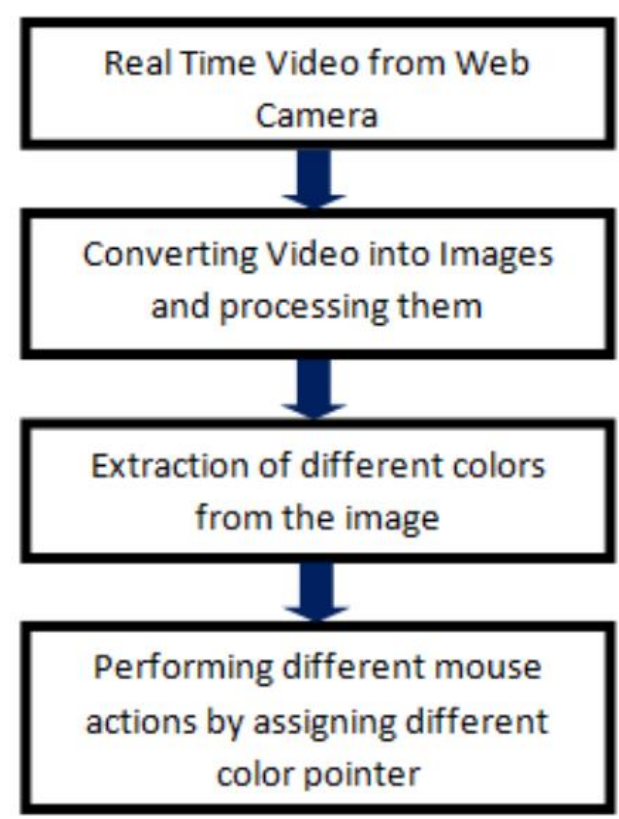

Fig. 2. The Basic Block Diagram

\section{ALGORITHUM}

Step 1. Capturing current video exploitation Web-Camera. Step 2. Process the individual video frame.

Step 3. Flipping of every video frame.

Step 4. Conversion of every frame to a gray scale video frame. Step 5. Color detection and extraction of the various color (RGB) from flipped gray scale video frame.

Step 6. Conversion of the finding video frame into a RGB to HSV color type.

Step 7. Finding the coordinates of the video frame and calculative its position.

Step 8. Pursuit the mouse pointer exploitation the coordinates obtained from the center of mass.

Step 9. Uniformly the single click and also the open click events of the mouse by distribution totally different color pointers.

\section{METHODOLOGY}

\section{Capturing the current video}

The digital camera of the computer is employed as a device. The digital camera catches the constant video at a settled casing rate and determination that is controlled by the instrumentality of the camera. The casing rate and determination will be modified within the framework if needed. Pc digital camera is employed to catch the important Time Video. Video is divided into video frame outlines in light-weight of 


\section{International Journal of Engineering Applied Sciences and Technology, 2020 Vol. 5, Issue 4, ISSN No. 2455-2143, Pages 295-299 \\ Published Online August 2020 in IJEAST (http://www.ijeast.com)}

the Federal Protective Service (Frames each second) of the camera process of individual Frames. $\mathrm{cam}=\mathrm{cv}$. VideoCapture $(0)$

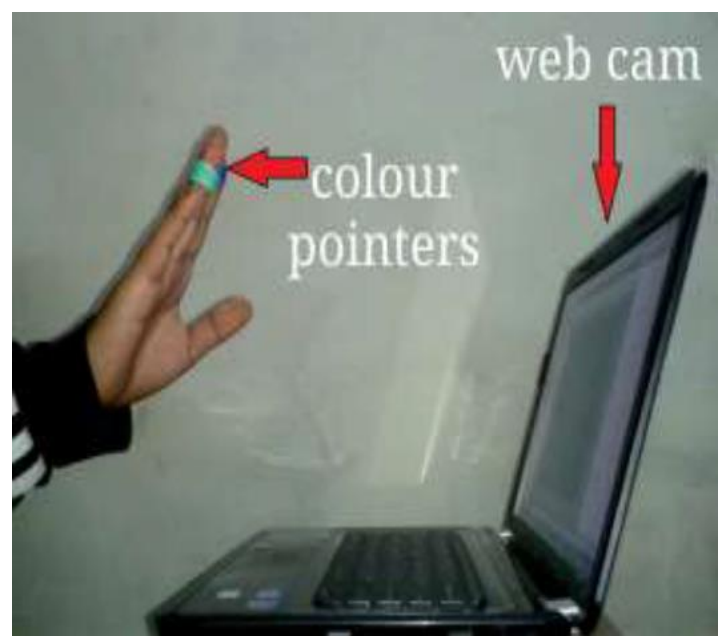

Fig. 3. Capturing Video

\section{Flipping ofvideo frames}

At the purpose once the camera catches a video frame, it's upset. This suggests on the off likelihood that we tend to move the shading pointer towards the left, the video frame of the pointer moves towards the privilege and also the different method around. It's sort of a video frame uninheritable once we stay before a mirror (Left is distinguished as right and right is recognized as cleared out). To take care of a strategic distance from this issue we've to vertically flip the video frame. \{The video frame the video frame \} caught could be a RGB picture and flipping activities cannot be foursquare performed thereon. That the individual shading channels of the video frame area unit isolated and afterwards they're flipped severally. Within the wake of flipping the red, blue and inexperienced hued channels individually, they're connected and a flipped RGB video frame is gotten.

frame $=$ cv.flip $($ frame, 1$)$

image_smooth $=$ cv.GaussianBlur $($ frame $,(7,7), 0)$

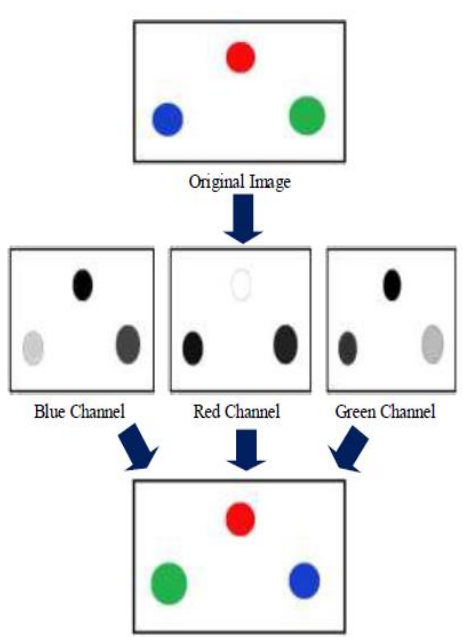

Fig. 4. Flipping of video frames

3. Conversion of Flippedvideo frame into Gray scalevideo frame

once contrasted with a shadedvideo frame, process many-sided quality is diminished in an exceedingly dim scalevideo frame. during this method the flippedvideo frame is modified over into a dim scalevideo frame. All the elemental operations were performed within the wake of adjusting over thevideo frame into dark scale.

mask $=$ np.zeros_like $($ frame $)$

image_roi $=$ cv.bitwise_and(image_smooth,mask)

image_hsv

cv.cvtColor(image_roi,cv.COLOR_BGR2HSV)

image_threshold=cv.inRange(image_hsv,lower_yello w,upper_yellow)

\section{Color Detection}

This can be the foremost important stride within the entire procedure. The red, inexperienced and blue shading article is recognized by subtracting the flipped shading smothered channel from the flipped Gray-Scale video frame. This makes a video frame that contains the recognized protest as a fix of dim encompassed by dark area.

contours, heirarchy $=c v$.findContours(image_threshold ,cv.RETR_TREE,cv.CHAIN_APPROX_NONE)

\section{Performing Clicking Actions}

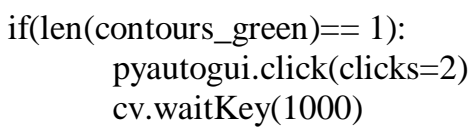


International Journal of Engineering Applied Sciences and Technology, 2020

Vol. 5, Issue 4, ISSN No. 2455-2143, Pages 295-299

Published Online August 2020 in IJEAST (http://www.ijeast.com)

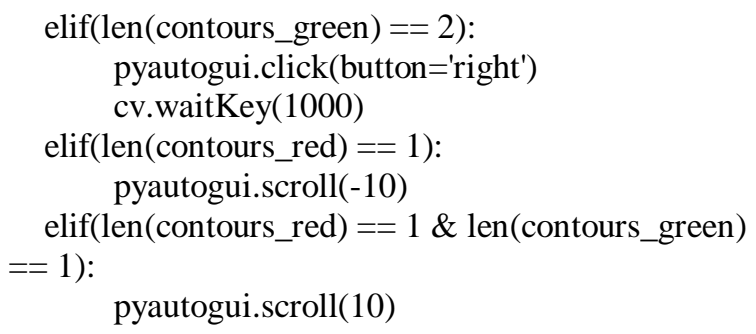

\section{CONCLUSION}

The process is employed to manage the mouse indicator and implement it's perform employing a period camera. We tend to enforced mouse movement, choice of the icons and its functions like right, left, double click and scrolling. This method is predicated on video frame comparison and motion detection technology to try and do mouse pointer movements and choice of icon. From the results, we are able to expect that if the algorithms can add all environments then our process will work a lot of expeditiously. This method may well be helpful in displays and to cut back work area. Within the future, we tend to attempt to add a lot of options like enlarging and shrinking windows, closing window, etc. by exploitation the palm and multiple fingers.

\section{REFERENCES}

1) Patil Nilesh, Sali Gaurav, Lokhande Nikhil, (2019), Mouse on Finger Tips using ML and AI, BE(CSE), JSPM's BSIOTR, Wagholi, Pune.

2) A. Erdem, E. Yardimci, Y. Atalay, V. Cetin, A. E. (2012) "Computer vision based mouse", Acoustics, Speech, and Signal Processing, Proceedings. (ICASS). IEEE International Conference.

3) Park Hojoon, (2013), “A Method for Controlling the Mouse Movement using a Real Time Camera", Brown University, Providence, RI, USA, Department of computer science.

4) Niyazi Kamran, Kumar Vikram, Mahe Swapnil, Vyawahare Swapnil, (2012), "Mouse Simulation Using Two Coloured Tapes", Department of Computer Science, University of Pune, India, International Journal of Information Sciences and Methods (IJIST) Vol.2, No.2.

5) Shah K N., Rathod K R. and Agravat S. J.,(2014) "A survey on Human Computer Interaction Mechanism Using Finger Tracking" International Journal of Computer Trends and Technology, 7(3), 174-177

6) Malik Shahzad, (2013) "Real-time Hand Tracking and Finger Tracking for Interaction", CSC2503F
7) Fethi Smach, Cedric Lemaître, Jean-Paul Gauthier Johel Miteran, Mohamed Atri 2008. "Generalized Fourier Descriptors with Applications to Objects Recognition in SVM Context", J Math Imaging Vis, Springer Science+Business Media 30: 43-71.

8) Vapnik, V.N, (1998), " The Statistical Learning Theory", Springer, Berlin.

9) P Robertson., R Laddaga., M Van Kleek,, (2004)” VR mouse vision based interface".

10) Banerjee Abhik, Ghosh Abhirup, Bharadwaj Koustuvmoni, (2014), " Mouse Control using a Web Camera based on Color Detection" ,IJCTT, vol.9.

11) Eckert, M. Lopez, M. ; Lazaro, C. ; Meneses, J. ; Martinez Ortega, J.F., (2015) Mokey - A motion based keyboard interpreter .Tech. Univ. of Madrid, Madrid, Spain.

12) Su, Xiaolin ,Zhang, Yunzhou ; Zhao, Qingyang ; Gao, Liang , (2015) VR keyboard: A humancomputer interaction device based on laser and video frame processing, VR keyboard: A humancomputer interaction device based on laser and video frame processing, College of Information Science and Engineering, Northeastern University, Shenyang, China

13) Jun Hu, Guolin Li, Xiang Xie, Zhong Lv, and Zhihua Wang, Senior Member, IEEE:Bare-fingers Touch Detection by the Button's Distortion in a Projector-Camera Process 
International Journal of Engineering Applied Sciences and Technology, 2020 Vol. 5, Issue 4, ISSN No. 2455-2143, Pages 295-299

Published Online August 2020 in IJEAST (http://www.ijeast.com) 